\title{
INTEGRATION OF AN INFORMATION SYSTEM TO SUGGEST A DRL MODEL IN RADIOLOGY DEPARTMENT AT AZADI HOSPITAL IN DUHOK-KURDISTAN REGION, IRAQ
}

\author{
SOLEEN JALADET Alsofi1 \\ Dept.. of Biomedical Engineering, College of Engineering, University of Duhok, KRI, Kurdistan \\ Region-Iraq
}

(Received: August 19, 2020; Accepted for Publication: September 20, 2020)

\begin{abstract}
Computer Tomography is a useful technique in diagnostic radiology. However, it emits high radiation doses which may cause harm to patients. For this, a quality management system, Diagnostic Reference Levels (DRL) is used in European countries. The aim of this study was to suggest a DRL system in Duhok Hospitals. Patient's data (70 - 80) were collected from Azadi Hospital for three main protocols head, chest and abdomen. Only 15 patients had complete data for calculations for the three protocols. The DRL results were then compared with the ones reported in several European countries. For example, in the head protocol for the a CTDI value of 81, the results of the calculated DLP and ED values were 1504 and 3.16 respectively. These values were found to be much higher than the corresponding values reported in Italy 13.12 and 2.76 respectively. As a result, the DRL using 75 th percentiles corresponding to these three dosimetric values were also high. Similar high results for the abdomen protocol were recorded, however, the results of the chest protocol showed to be within the average range. Part two of this work involved designing a Flow Chart based on an Information System to help running the CT scan procedure more effectively. Additionally, an extra repository system was added to this model to solve the short capacity issue in Duhok Hospitals.
\end{abstract}

KEYWORDS: Data Management Systems, Data Repository, Diagnostic Reference Level, Dose Management, Information Technology, Radiology Information System

\section{INTRODUCTION}

$\mathbf{O}$ ne of the great challenges that may face today's Hospitals in many countries is the issue of gathering, storing, tracing and maintaining data in regard to patient information (Kruse et. al., 2016). Due to the advancements in Information Technology (IT) several techniques have been implemented in order to enhance the daily workflow in a hospital ( Sawaneh et. al., 2018). These digital transformations include improved Wireless Networks, Cloud Solutions, Data Management Systems as well as many other software which their applications may help running the medical devices that are available in several departments of a hospital. Implementing such Information System will lead to the improvements and contribute into a collective system which enables hospitals to work more efficiently such as the Hospital Information System (HIS) (Balaraman, and Kosalram, 2013). Although HIS was primarily used for financial purposes and only partially for patient care systems, later it became more complex as many hospitals incorporated these specialized systems in several departments (Chatzoglou et. al., 2012) (Mehdipour, and Zerehkafi, 2013) . One of the main departments of a hospital where these digital systems are applied are the radiological departments. They use the data (patient information) which is stored in the HIS, moreover these departments use images created from medical devices such as X-Ray, Computed Tomography, Ultrasound, and Magnetic Resonance Imaging etc. which contain valuable data. This leads to the necessity that a radiological department needs to have its own Information System alongside with the HIS which is internationally known as a Radiology Information System (RIS) (Babić et. al., 2012). RIS is a database designed to support operational workflow and medical analysis within a radiology department. This repository stores patients' personal data and exams carried out, but it has only limited capacity in storing images and dosimetric values which are obtained from 
these examinations (Radiologists, 2008). This leads to the issue that images are lost in some cases hence would result in a patient's reexamination and would also mean to expose the patient to higher radiation dose (Palorini et. al., 2014) (Nitrosi et. al., 2014) . Dosimetric information, together with RIS information such as patient data and report outcome could contextualize the dose and consequently help in optimizing the image quality. In addition, dosimetric value information will be stored in the additional database allowing further real patient centered dosimetric value optimization and treatment which enhances clinical accuracy for patients undergoing many ionizing procedures (Palorini et. al., 2014) (Nitrosi et. al., 2014). However, there is another issue to be solved which is, due to the limited capacity of RIS in storing images. To overcome this issue, there are several other software solutions that run alongside the RIS. These techniques will not only solve the limited storage capacity of RIS but instead will avoid unnecessary or unproductive radiation exposure.

One of these techniques is the introduction of the Diagnostic Reference Levels (DRL) which was implemented by the International Commission on Radiological Protection (ICRP) in 1991 (COMMISSION, Radiation protection $\mathrm{N}^{\circ} 185,2018$ ). to ensure adequate doses that a patient may receive in the different diagnoses examinations, a quality management system should be employed (COMMISSION, Radiation protection $\mathrm{N}^{\circ} 185,2018$ ). And as a part of the quality management in many countries DRLs has been established in 1997 and was introduced to the European legislation by the Medical Exposure Directive 97/43/EURATOM (COMMISSION, Radiation protection $\mathrm{N}^{\circ} 185$, 2018) This was established in order to ensure that doses to which patients are subjected are as low as reasonably possible. However, it is important to mention that DRLs are not dose limits but more of a guide value, mostly utilized to analyze imaging methods which normally result in extremely high patient doses and thus should be adjusted and optimized accordingly (COMMISSION, Radiation protection $\mathrm{N}^{\circ} 185$, 2018).

Computed Tomography (CT) as one of the useful techniques in diagnostic radiology has a very extensive scope of clinical functions and since due to the CT scan high radiation dose it is a subject of interest for many institutions, therefore internationally it relies heavily on
DRLs (Bosch de Basea et. al., 2015). Since it is generally considered that dose radiation, regardless of the amount, is able to harm the patient, the CT's high radiation dose are a subject of interest for many institutions (Kavanagh et. al. , 2018) (Pearce et. al., 2012).

It has been noticed that hospitals and health institutes in Duhok/KRG - Iraq does not have any DRL standards as well as the mentioned additional repository to store the images and the dosimetric values. Meaning, that Radiologists and Physicians work very hard to manage the examinations without any standards, where they only estimate the patient's dose amount based on their knowledge and experience. Furthermore, in many times they also have to repeat examinations since many patients lose their images and thus have to be re-examined. For these reasons and others, it is not only insufficient and inaccurate; this creates an unnecessary financial burden on the hospitals and it also raises harm for patients since too much radiation can cause severe health risks. Therefore, the aim of this paper was to suggest a DRL model as well as to recommend an additional repository system which provide hospitals in Duhok the necessary platform to manage patient data including images in order to improve the patient's safety as well as helping hospitals and health institutes optimizing the dose radiation management. As a model this paper has taken several CT Scan examples from the Azadi Hospital in Duhok to suggest some possible DRL standards in order to adjust more to the European healthcare enterprise by improving quality and safety.

\section{MATERIALS AND METHODS}

The CT scan device model Toshiba 64 slice with its software system version "Aquilion V4.61ER004" is currently used in the Azadi Hospital with the company set up configuration. This CT scan system include the standard protocols for different body parts (Head, Neck, Chest, Abdomen, Pelvis, Leg, Chest-to-Pelvis). In this work, all use-cases (samples) were focused primarily on the most used protocols in the Azadi Hospital which included head, chest and abdomen CT examinations. All needed data for this work were self-collected during personal attendance at the CT scan examination sessions. A total number of $70-80$ patient data were collected. However, complete CT performance information and examination parameters that 
served the purpose of this work, were only obtained from 15 patients as shown in Table 1. In order to process and store these data, an integrated development environment (IDE), such as Visual Studio or Eclipse is needed. The program and script languages which are recommended are SQL, C\# and Python.

Table (1): CT Examination Parameters from Azadi Hospital

\begin{tabular}{|c|c|c|c|c|c|c|c|c|c|c|c|}
\hline Name & Gender & Age & Body Part & Scan Mode & Tube Potential (kV) & \begin{tabular}{|c|}
$\begin{array}{c}\text { Tube Current } \\
(\mathrm{mA})\end{array}$ \\
\end{tabular} & \begin{tabular}{|c|}
$\begin{array}{c}\text { Rotation } \\
\text { Time }\end{array}$ \\
\end{tabular} & Total Scan Time & Slice Thickness & CTDI $_{\text {vol }}$ (mGy) & DLP (mGy.cm) \\
\hline Patient 1 & $\mathrm{~F}$ & 42 & Head & Brain HCT (Helical) & 120 & 300 & 0.75 & 18.09 & $0.5 \times 32$ & 80.5 & 1503.6 \\
\hline Patient 2 & M & 70 & Head & Brain HCT (Helical) & 120 & 300 & 0.75 & 18.44 & $0.5 \times 32$ & 80.8 & 1544 \\
\hline Patient 3 & M & 54 & Head & Brain HCT (Helical) & 120 & 300 & 0.75 & 17.76 & $0.5 \times 32$ & 80.3 & 1499.4 \\
\hline Patient 4 & $\mathrm{~F}$ & 49 & Head & Brain HCT (Helical) & 120 & 300 & 0.75 & 17.73 & $0.5 \times 32$ & 80 & 1463.2 \\
\hline Patient 5 & M & 50 & Head & Brain HCT (Helical) & 120 & 300 & 0.75 & 17.98 & $0.5 \times 32$ & 80.8 & 1491 \\
\hline Patient 6 & M & 71 & Chest & Chest HCT (Helical) & 120 & 268 & 0.5 & 19.45 & $0.5 \times 64$ & 9.9 & 402.9 \\
\hline Patient 7 & $\mathrm{~F}$ & 66 & Chest & Chest HCT (Helical) & 120 & 350 & 0.5 & 18.77 & $0.5 \times 64$ & 6.5 & 292.6 \\
\hline Patient 8 & M & 54 & Chest & Chest HCT (Helical) & 120 & 281 & 0.5 & 18.96 & $0.5 \times 64$ & 8 & 373.8 \\
\hline Patient 9 & M & 66 & Chest & Chest HCT (Helical) & 120 & 305 & 0.5 & 19.12 & $0.5 \times 64$ & 6.5 & 292.6 \\
\hline Patient 10 & M & 73 & Chest & Chest HCT (Helical) & 120 & 264 & 0.5 & 21.22 & $0.5 \times 64$ & 10.9 & 390.7 \\
\hline Patient 11 & $\mathrm{~F}$ & 60 & Abdomen & Abdomen-C HCT (Helical) & 120 & 460 & 0.5 & 19.55 & $0.5 \times 64$ & 35 & 1581.2 \\
\hline Patient 12 & $\mathrm{~F}$ & 29 & Abdomen & Abdomen-C HCT (Helical) & 120 & 403 & 0.5 & 18.98 & $0.5 \times 64$ & 30.6 & 1020.3 \\
\hline Patient 13 & $\mathrm{~F}$ & 34 & Abdomen & Abdomen-C HCT (Helical) & 120 & 500 & 0.5 & 19.61 & $0.5 \times 64$ & 38 & 2036.1 \\
\hline Patient 14 & M & 71 & Abdomen & Abdomen-C HCT (Helical) & 120 & 328 & 0.5 & 18.71 & $0.5 \times 64$ & 24.2 & 1646.1 \\
\hline Patient 15 & $\mathrm{~F}$ & 56 & Abdomen & Abdomen-C HCT (Helical) & 120 & 425 & 0.5 & 19.42 & $0.5 \times 64$ & 32.5 & 1245 \\
\hline
\end{tabular}

To start, the patients had to give his/her personal information to the HIS which contained name, age, gender, weight, etc. The HIS then assigned a Patient ID that will function as the unique identifier. After that the patient normally is send according to the diagnosis to a medical device (e.g. CT scan) which created an image of the correspondent body part as shown in the diagram of Figure 1. 


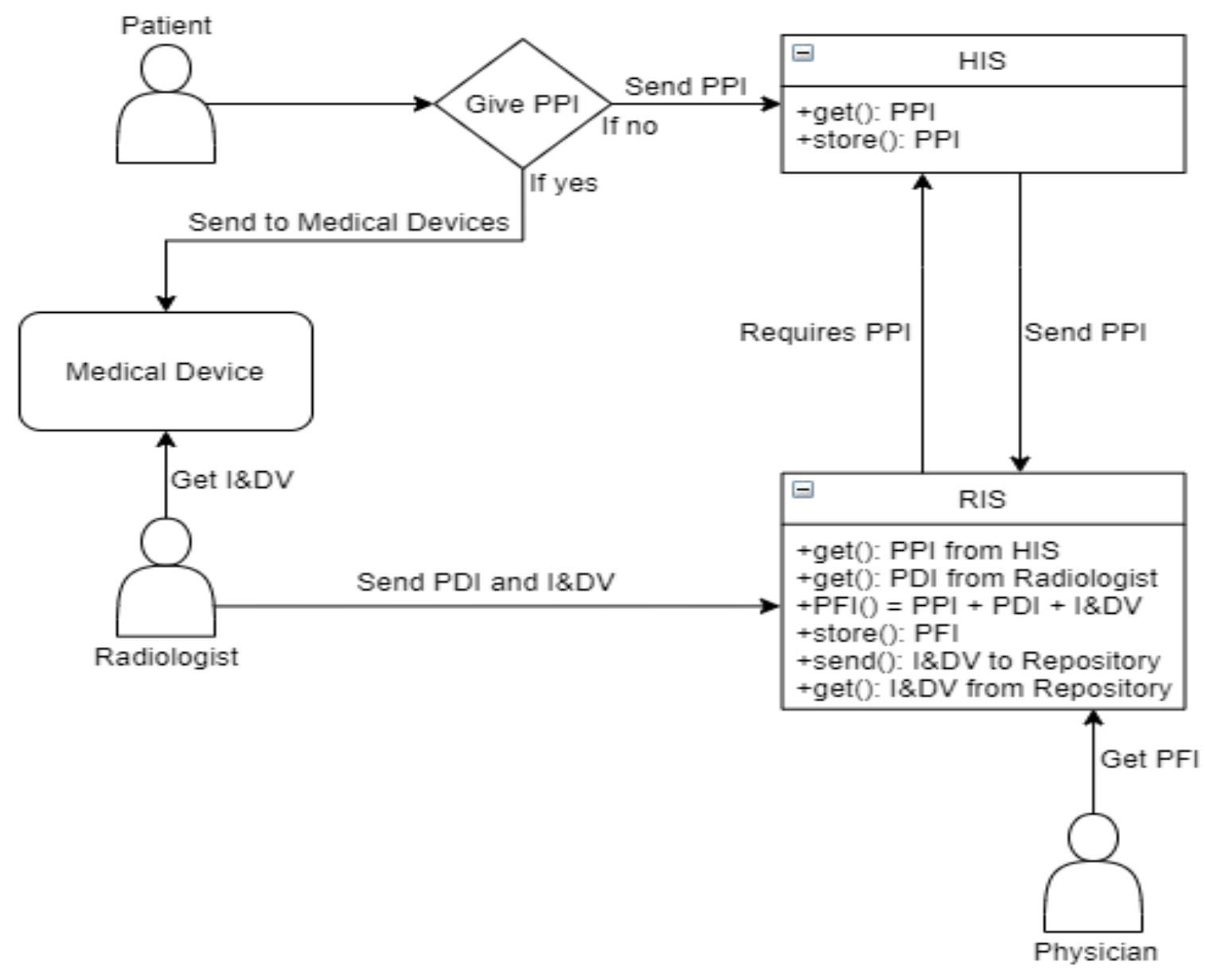

Fig. (1): Flow Chart Diagram developed to display a typical use-case scenario in the hospital

HIS = Hospital Information System / RIS = Radiology Information System / PPI = Patient Personal Information / PDI $=$ Patient Detailed Information / PFI = Patient Full Information / I = Imagery / DV = Dosimetric Values / Medical Devices can include: X-Ray, Computed Tomography, Magnetic Resonance Imaging, Ultrasound etc.

In addition, the multi-detector CT scanner recorded several data, such as the dosimetric values. The three main dose indices are Computed Tomography Dose Index volume $\left(\mathrm{CTDI}_{\mathrm{vol}}\right)$ measured in milligrays (mGy), the Dose Length Product (DLP) measured in milligrays per centimeter $(\mathrm{mGy}-\mathrm{cm})$ and the Effective Dose (ED) measured in millisieverts $(\mathrm{mSv})$ as was shown in Table 1 (Granata et. al., 2014).

However, storing these values together with the imagery was proven to be an issue since the available storage space was not enough to contain all these documents, which lead to the fact that patients had to carry their x-rays with them each time they had to revisit the hospital, thus an additional repository was needed in order to fully store all relevant patient documents.

As mentioned above the CT scanner has been configured by the suppliers and it seemed that it has been limited due to the company's policy in addition, to unavailability of necessary software and thus, a number of dosimetric values were not shown. Therefore, to determine all the values needed in order to set a possible DRL standard as well as revealing the difference in the results between patients using the same body part, a number of calculations were carried out. One of these values to be calculated was the estimated length $(\mathrm{L})$ measured in centimeter $(\mathrm{cm})$ which was based on the CTDI ${ }_{\mathrm{vol}}$ and DLP generated by the CT scanner according to the following equation (Ekpo et. al., 2018) (Saravanakumar et. al., 2014).

$D L P=C T D I_{\text {vol }} \times L$

The second value to be calculated was the Effective Dose (ED) which represents the equivalent whole-body dose according to the 
following equation (Deak et. al., 2010) (Ekpo et. al., 2018)

$E D=D L P \times k$

Where $\mathrm{k}$ is the tissue weighting factor based on the scanned body region. Conversion factors for adults of various ages are shown in Table 2 as described and recommended in the ICRP publication 60 \& 103 (Eckerman et. al., 2012) (VALENTIN, 2007) (Kobayashi et. al., 2019).

Table (2): Conversion Factors for adults

\begin{tabular}{|l|c|}
\hline Scanned body parts & k (tissue weighting factor) \\
\hline Head / Neck & 0.0031 \\
\hline Head & 0.0021 \\
\hline Neck & 0.0059 \\
\hline Chest & 0.014 \\
\hline Abdomen & 0.015 \\
\hline Trunk & 0.015 \\
\hline
\end{tabular}

The values of $\mathrm{CTDI}_{\mathrm{vol}}$, DLP, L and ED for each examination sample were calculated and recorded. These value calculations and dosimetry technique was based on the methods proposed by European Guidelines and also according to the conditions and recommendations of the ICRP (VALENTIN, 2007) (COMMISSION, 2000) (Saravanakumar et. al., 2014). Additional values that were calculated in this work, was to determine the percentiles of the three dosimetric values. It is important to mention that image quality improves proportional with the increasing percentage of dose radiation and vice versa. Therefore, the most commonly used percentile as an indicator in Europe to do the DRL calculations for each examination are $25^{\text {th }}, 50^{\text {th }}$ and $75^{\text {th }}$ percentile (Ekpo et. al., 2018) (Nakada Y et. al., 2018) (Saravanakumar et. al., 2014)

While the $25^{\text {th }}$ percentile values are normally used to assess the lowest dose levels for the price of significantly deteriorated image quality, the $50^{\text {th }}$ percentile on the other hand, functions as median and provides dose levels that institutes should strive towards to. However, the $75^{\text {th }}$ percentile values are also the most effective when establishing the DRL function also as proposed dose values which should not be exceeded (VALENTIN, 2007). Most European countries use the $75^{\text {th }}$ percentile values thus this work will estimate the DRL values using the same percentile as the European countries do in order to be able to compare them with each other (Health, 2018) (Richard Veit, and Burkhard Bauer, 2019).

\section{Development of a DRL model}

In this work, the $75^{\text {th }}$ percentile of the dosimetric values CTDI $_{\text {vol }}$, DLP and ED were then used to calculate the DRL values. Then, these new calculated DRL values were compared with the ones of a number of European countries to identify whether the obtained data exceeded their limits and thus to suggest the needs for adjustment. Finally, a further developed flow chart was designed to integrate an Information System model.

\section{RESULTS AND DISCUSSION}

The results of the first step which involved the calculation of length $\mathrm{L}$, are summarized in Table 3. These results were obtained by extracting the dosimetric values from the additional database via the RIS. 
Table (3): Dosimetric Values and Patient's Length

\begin{tabular}{|l|c|c|c|}
\hline Name & CTDI $_{\text {vol }}$ (mGy) & DLP (mGy.cm) & Length (cm) \\
\hline Patient 1 & 80.5 & 1503.6 & 18.68 \\
\hline Patient 2 & 80.8 & 1544 & 19.11 \\
\hline Patient 3 & 80.3 & 1499.4 & 18.67 \\
\hline Patient 4 & 80 & 1463.2 & 18.29 \\
\hline Patient 5 & 80.8 & 1491 & 18.45 \\
\hline Patient 6 & 9.9 & 402.9 & 40.70 \\
\hline Patient 7 & 6.5 & 292.6 & 45.02 \\
\hline Patient 8 & 8 & 373.8 & 46.73 \\
\hline Patient 9 & 6.5 & 292.6 & 45.02 \\
\hline Patient 10 & 10.9 & 390.7 & 35.84 \\
\hline Patient 11 & 35 & 1581.2 & 45.18 \\
\hline Patient 12 & 30.6 & 1020.3 & 33.34 \\
\hline Patient 13 & 38 & 2036.1 & 53.58 \\
\hline Patient 14 & 24.2 & 1646.1 & 68.02 \\
\hline Patient 15 & 32.5 & 1245 & 38.31 \\
\hline
\end{tabular}

From this Table it can be noticed that $\mathrm{L}$ varies within the same protocol, where the patient samples from 1 to 5 represented the Head protocol, the patient samples from 6 to 10 represented the Chest protocol and the patient samples 11 to 15 represented the Abdomen protocol. For example, the two main dosimetric values for Patient 1 were obtained by the CT scanner with $80.5 \mathrm{mGy}$ and $1503.6 \mathrm{mGy} . \mathrm{cm}$ respectively. Then, by applying equation (1), the estimated L was indicated $18.68 \mathrm{~cm}$ and all the other samples followed the same procedures.

The shown result in the above Table indicated, that the observed amount of the DLP value increased proportional with the raising length $\mathrm{L}$, which led to the fact that the amount of the DLP could be controlled and limited by the radiologist/physician depending on the length of the body part that was selected and estimated (BUSHBERG et. al., 2012).

The results of the following step represented the Effective Dose (ED) measured by applying equation (2) with the use of the $\mathrm{k}$ factor for the Head was 0.0021 , for the Chest was 0.014 and for the Abdomen was 0.015 where all results are represented in Table 4.

Table (4): The Effective Dose for each Patient

\begin{tabular}{|l|c|c|c|}
\hline Name & DLP (mGy.cm) & K Factor & ED (m Sv) \\
\hline Patient 1 & 1503.6 & 0.0021 & 3.16 \\
\hline Patient 2 & 1544 & 0.0021 & 3.24 \\
\hline Patient 3 & 1499.4 & 0.0021 & 3.15 \\
\hline Patient 4 & 1463.2 & 0.0021 & 3.07 \\
\hline Patient 5 & 1491 & 0.0021 & 3.13 \\
\hline Patient 6 & 402.9 & 0.014 & 5.64 \\
\hline Patient 7 & 292.6 & 0.014 & 4.10 \\
\hline Patient 8 & 373.8 & 0.014 & 5.23 \\
\hline Patient 9 & 292.6 & 0.014 & 4.10 \\
\hline Patient 10 & 390.7 & 0.014 & 5.47 \\
\hline Patient 11 & 1581.2 & 0.015 & 23.72 \\
\hline Patient 12 & 1020.3 & 0.015 & 15.30 \\
\hline Patient 13 & 2036.1 & 0.015 & 30.54 \\
\hline Patient 14 & 1646.1 & 0.015 & 24.69 \\
\hline Patient 15 & 1245 & 0.015 & 18.68 \\
\hline
\end{tabular}

The results revealed that the ED amount also increased proportional to the amount of the DLP, which described the dose amount that each patient received during the scanning (Ekpo et. al., 2018) (Huda et. al., 2008).

According to the results shown in Table 3 and 4, it was possible to consider that the selected length played the main role in the dose amount that was given to any patient. From these results it appeared that both, the length and the ED in most cases are higher in comparison to other countries, meaning that a higher length correlated to a higher dose amount thus exposing patients to a greater harm of radiation.

The result of different samples showed how the used method could help identify where 
protocols are probably not standardized and to suggest where further optimization actions should be taken. The factors that affect and help to establish the DRL are CTDI ${ }_{\text {vol }}$, DLP and ED (Ekpo et. al., 2018) (Saravanakumar et. al., 2014).

Actually, all three factors are routinely used for comparing exposures from different scanning protocols, for setting diagnostic reference levels (DRLs), and for dose optimization. DRLs allow radiology departments to compare their dose levels with regional or national standards. These levels are expected not to be exceeded for standard procedures when good and normal practice regarding diagnostic and technical performance is applied (Granata et. al., 2014).

\section{Development of a DRL Model}

In order to design a DRL model in our hospitals, the dosimetric values were used to determine the commonly used percentiles $\left(25^{\text {th }}\right.$, $50^{\text {th }}$ and $75^{\text {th }}$ ) applied by the European countries for all three protocols as shown in Table 5. The importance of providing these data, is for any further studies that wishes to develop a DRL model using any of these percentiles.

Table (5): Percentiles of Dosimetric Values

\begin{tabular}{|l|c|c|c|c|c|c|c|c|c|}
\hline \multirow{2}{*}{ Body region } & \multicolumn{3}{|c|}{$\begin{array}{c}\text { CTDIvol (mGy) } \\
\text { Percentile values }\end{array}$} & \multicolumn{3}{c|}{$\begin{array}{c}\text { DLP (mGy.cm) } \\
\text { Percentile values }\end{array}$} & \multicolumn{3}{c|}{$\begin{array}{c}\text { Effective Dose } \\
\text { (mSv) Percentile } \\
\text { values }\end{array}$} \\
\cline { 2 - 10 } & $25^{\text {th }}$ & $50^{\text {th }}$ & $75^{\text {th }}$ & $25^{\text {th }}$ & $50^{\text {th }}$ & $75^{\text {th }}$ & $25^{\text {th }}$ & $50^{\text {th }}$ & $75^{\text {th }}$ \\
\hline Head & 00.3 & 80.5 & 80.8 & 1491 & 1499 & 1504 & 3.13 & 3.15 & 3.16 \\
\hline Chest & 6.5 & 8 & 9.9 & 292.6 & 373.8 & 390.7 & 4.1 & 5.23 & 5.47 \\
\hline Abdomen & 30.6 & 32.5 & 35 & 1245 & 1581 & 1646 & 18.68 & 23.72 & 24.69 \\
\hline
\end{tabular}

The DRL standards were then calculated by using the most frequently used percentiles $75^{\text {th }}$ of the dosimetric values, a value that is used by most of the European countries. These calculations were made according to the European Guidelines and also according to the conditions and recommendations of the ICRP (VALENTIN, 2007) (COMMISSION, 2000) (Saravanakumar et. al., 2014).
As mentioned before, the selected length could be considered as the main role in the dose amount that was given to any patient. Therefore, the European countries set an average length for each specific part of the body region. Thus, in this study it was recommendable to do so as well. Using the $75^{\text {th }}$ percentile, the length values for the samples have been calculated by measuring the ratio of the two dosimetric values DLP and CTDI $_{\mathrm{vol}}$ as shown in Table 6 .

Table (6): Length values using $75^{\text {th }}$ percentile

\begin{tabular}{|l|c|c|c|}
\hline Body region & CTDlvol (mGy) & DLP (mGy.cm) & L (DLPICTDI)(cm) \\
\hline Head & 80.8 & 1503.6 & 18.61 \\
\hline Chest & 9.9 & 390.7 & 39.46 \\
\hline Abdomen & 35 & 1646.1 & 47.03 \\
\hline
\end{tabular}

The results of Table 6 made clear that the length of the Head with $18.61 \mathrm{~cm}$, Chest with $39.46 \mathrm{~cm}$ and Abdomen with $47.03 \mathrm{~cm}$ were exceeding the ones from other European countries. For example, in Germany as one of the leading countries in this domain the average length for the Head, Chest and Abdomen region is almost $14.17,35$ and $24 \mathrm{~cm}$ respectively (Stamm et. al., 2017).

Most European countries use the $75^{\text {th }}$ percentile values due to the fact that DRLs should be made accessible for common studies with commensurate dose values. Therefore, collaborations between authorities and professional associations have been established with the purpose of creating national DRLs by using the $75^{\text {th }}$ percentile of the examined distribution of patient doses in their countries (Health, 2018) (Richard Veit, and Burkhard Bauer, 2019).

It is important to mention that these DRL values cannot be considered as standard values 
unless they are in a suitable range of other reported DRL ranges. Therefore, these study values were further compared with other standardized DRL values from several selected European countries as shown in Table 7.

Table (7): Dosimetric values of selected European countries

\begin{tabular}{|l|c|c|c|c|c|c|c|c|c|}
\hline \multicolumn{1}{|c|}{ Body region } & \multicolumn{3}{|c|}{ Head } & \multicolumn{3}{c|}{ Chest } & \multicolumn{3}{c|}{ Abdomen } \\
\hline Dosimetriv values & CTDIvol & DLP & ED & CTDlvol & DLP & ED & CTDIvol & DLP & ED \\
\hline This study & 81 & 1504 & 3.16 & 10 & 391 & 5.47 & 35 & 1646 & 24.69 \\
\hline Germany & 60 & 850 & 1.79 & 10 & 350 & 4.90 & 15 & 360 & 5.40 \\
\hline Sweden & 60 & 1000 & 2.10 & 9 & 350 & 4.90 & 11 & 550 & 8.25 \\
\hline Italy & 69 & 1312 & 2.76 & 15 & 569 & 7.97 & 18 & 550 & 8.25 \\
\hline Slovenia & 56 & 865 & 1.82 & 13 & 420 & 5.88 & 16 & 550 & 8.25 \\
\hline Czech Republic & 65 & 1100 & 2.31 & 15 & 500 & 7.00 & 19 & 750 & 11.25 \\
\hline Belgium & 50 & 900 & 1.89 & 8 & 260 & 3.64 & 10 & 490 & 7.35 \\
\hline France & 58 & 1050 & 2.21 & 20 & 500 & 7.00 & 25 & 650 & 9.75 \\
\hline Bulgaria & 60 & 1000 & 2.10 & 16 & 500 & 7.00 & 18 & 470 & 7.05 \\
\hline Poland & 60 & 1050 & 2.21 & 30 & 650 & 9.10 & 35 & 780 & 11.70 \\
\hline Finland & 55 & 800 & 1.68 & 9 & 290 & 4.06 & 12 & 560 & 8.40 \\
\hline
\end{tabular}

The values in Table 7 display the CTDI $_{\mathrm{vol}}$, DLP and ED range domain for Head, Chest and Abdomen. The range domain was determined from the lowest to the highest value for all selected European countries. This was valid for $\mathrm{CTDI}_{\mathrm{vol}}$, DLP and ED. For example, in the Head protocol, the $\mathrm{CTDI}_{\mathrm{vol}}$ value obtained in this study was 81 while the lowest $\mathrm{CTDI}_{\mathrm{vol}}$ value for the same protocol reported in Belgium was 50 and the highest value reported in Italy was 69 . This made clear that the determined value of this study of 81 was exceeding the range domain compared to the selected European countries. The DLP value for the Head protocol obtained in this study was 1504 . This value also exceeded the range domain in the selected countries where the lowest DLP value in Finland was 800 and the highest value in Italy was 1312. As for the ED, the value of the Head protocol of this study was 3.16 while the lowest value reported in Finland was 1.68 and the highest value reported in Italy was 2.76. As a result, the correspondent DRL values of this study were also high due the three dosimetric values. However, it is interesting to mention that the result of the Chest area showed that it is well within the middle of the range domain of the selected countries. The results of the dosimetric values for Abdomen from this study were also very high compared to the ones from the other countries. Thus, standard limitations are needed in order to be able to reduce the patient's dose amount. A more detailed range display from the lowest to the highest values of CTDI ${ }_{\mathrm{vol}}$, DLP and ED within all three protocols Head, Chest and Abdomen is shown in Figure 2. 


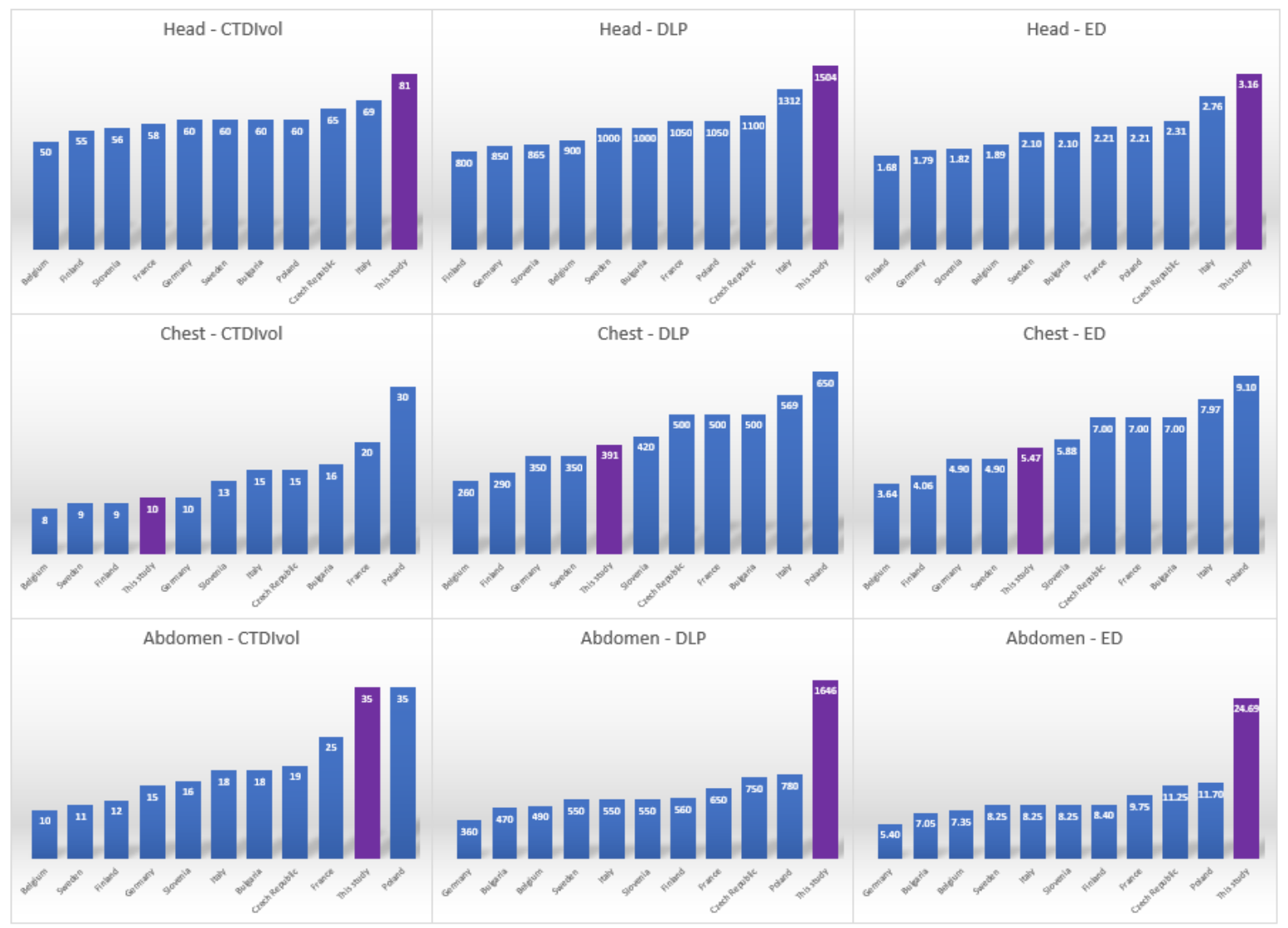

Fig. (2): Dosimetric value range domain for Head, Chest and Abdomen

The results of the Length obtained in this study for the three protocols were also compared to the selected European countries as displayed in Table 8.

Table (8): Length Comparison

\begin{tabular}{|l|c|c|c|}
\hline \multicolumn{1}{|c|}{ Body region } & Head & Chest & Abdomen \\
\hline Dosimetriv values & L (DLP/CTDI) & L (DLP/CTDI) & L (DLPICTDI) \\
\hline This study & 18.57 & 39.10 & 47.03 \\
\hline Germany & 14.17 & 35.00 & 24.00 \\
\hline Sweden & 16.67 & 38.89 & 50.00 \\
\hline Italy & 19.01 & 37.93 & 30.56 \\
\hline Slovenia & 15.45 & 32.31 & 34.38 \\
\hline Czech Republic & 16.92 & 33.33 & 39.47 \\
\hline Belgium & 18.00 & 32.50 & 49.00 \\
\hline France & 18.10 & 25.00 & 26.00 \\
\hline Bulgaria & 16.67 & 31.25 & 26.11 \\
\hline Poland & 17.50 & 21.67 & 22.29 \\
\hline Finland & 14.55 & 32.22 & 46.67 \\
\hline
\end{tabular}

A more detailed range display from the lowest to the highest Length within all three protocols Head, Chest and Abdomen is shown in Figure 3. 


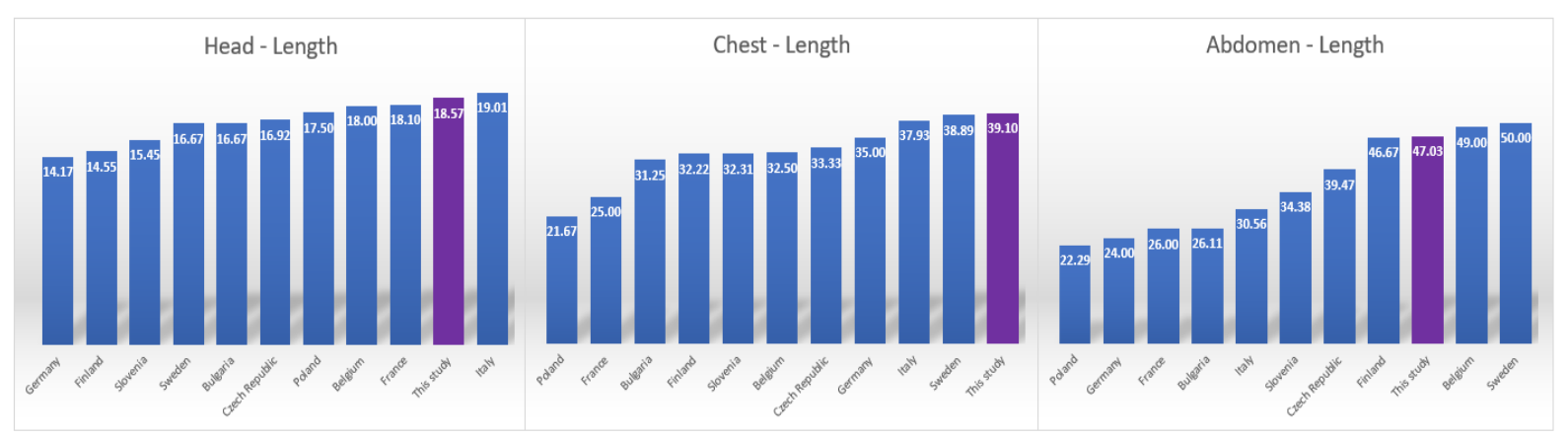

Fig. (3): Detailed Length Display

Analyzing this figure, the following notes may generally be extracted. For example, while the dosimetric values for Chest are within average range domain, the Length is only a little higher compared with the other countries and therefore could be reduced. The real problematic case was reflected in the Head and Abdomen protocol since the dosimetric values and the
Length were very high. A dose amount should be adjusted, by implementing a suggested DRL.

With help of this new designed DRL model, a new control mechanism model was constructed which functioned as an interface between the RIS and the Radiologist/Physician as shown in Figure

4.

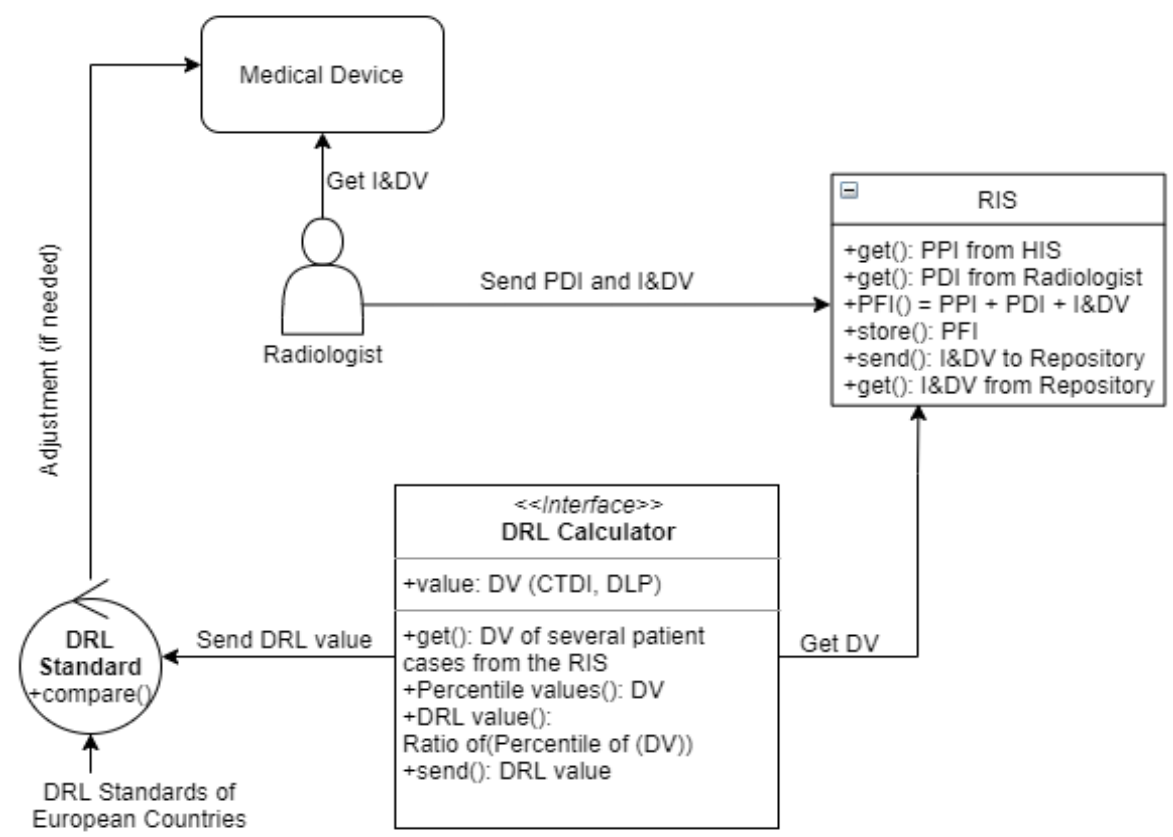

Fig. (4): Suggested DRL Control Model

Implementing this model, the interface will send the DRL value to the control unit "DRL Standard" which then evaluated it by comparing them with the standard values of the European countries. If the DRL value exceeded the European standard values, then the Radiologist/Physician is asked by the system, more precisely by the software which runs the CT scanner, to adjust the estimated length, since the results made clear that the selection of a body region's length has a direct impact of the dose amount. However, if the calculated DRL value was within the European standard values, then the radiologist/physician could continue with the process.

A final Flow Chart as shown in Figure 5 was designed, which may run the CT scan procedure effectively as well as control and suggest adjustments 
of the dose amount. It is worth mentioning that an should help in solving the short storage capacity of extra repository system was added to the model that the RIS as mentioned previously.



Fig. (5): Enhanced Flow Chart Diagram of a typical use-case scenario in the hospital

To this end, integrating the new Information System Model will improve the benefit of such important medical equipment and on the same time minimizing the effects of harmful and unnecessary doses of radiation that a patient may be exposed to while it may also prevent unnecessary financial expenditure for the hospital.

\section{CONCLUSION}

This work involved the integration of an Information System for suggesting a DRL model that may ensure the safety of a patient by implementing two solution methods. The first was to set an additional repository to overcome the capacity limitations of the hospital's data storage. The second solution was to create a safe radiation management system through suggesting a DRL model at the CT scan unit in Azadi hospital.

The results revealed that the dosimetric values were much higher than those internationally reported. Therefore, from these findings it may be concluded that such an Information System is of a necessity for patient's safety as well as for supporting the Radiologists/Physicians to perform radiological examinations in a safer manner in addition; to reducing financial costs and improvement of the hospital daily workflow.

\section{ACKNOWLEDGEMENT}

The author gratefully acknowledge the contributions of the Radiology Department as well as the hospital staff from the Azadi Hospital of Duhok, Kurdistan Region - Iraq. 


\section{REFERENCES}

A. Nitrosi, A. Corazza, M. Bertolini, R. Sghedoni, P. Pattacini, and M. Iori. (2014). Patient dose management solution directly integrated in the RIS: "Gray Detector" software. Springer, 27(6). doi:10.1007/s10278-014-9715-y

A. Saravanakumar, K. Vaideki, K. N. Govindarajan, and S. Jayakumar. (2014). Establishment of diagnostic reference levels in computed tomography for select procedures in Pudhuchery, India. Journal of Medical Physics. doi:10.4103/0971-6203.125509

- Claudio Granata, Daniela Origgi, Federica Palorini, Domenica Matranga, and Sergio Salerno. (2014). Radiation dose from multidetector CT studies in children: results from the first Italian nationwide survey. Springer. doi:10.1007/s00247-014-3201-z

COMMISSION, E. (2000). European guidelines on quality criteria for computed tomography. Brussels: European Commission.

Ernest U Ekpo, Thomas Adejoh, Judith D Akwo, Owujekwe C Emeka, Ali A Modu, Mohammed Abba, Kudirat A Adesina, David O Omiyi and Uche H Chiegwu. (2018). Diagnostic reference levels for commoncomputed tomography (CT) examinations: results from the first nigerian nationwide dose survey. Journal of Radiological Protection.

EUROPEAN COMMISSION. (2018). Radiation protection $\mathrm{N}^{\circ}$ 185. Luxembourg: European Union. Retrieved from http://www.eurosafeimaging.org/wp/wpcontent/uploads/2018/09/rp_185.pdf

Federica Palorini, Daniela Origgi, Claudio Granata, Domenica Matranga, and Sergio Salerno. (2014). Adult exposures from MDCT including multiphase studies: First italian nationwide survey. National Center for Biotechnology Information (NCBI). doi:10.1007/s00330-013-3031-7

BIBLIOGRAPHY G. Stamm, H. Lenzen, M. Wucherer, G. Brix, A. Schegerer, A. Sommer, Institute for Diagnostic and Interventional Radiology Göttingen/DE, Institute of Clinical Radiology Münster/DE, Institut für Medizinische Physik Nürnberg, D/DE, Neuherberg/DE, 2017. 2nd Modification and Update of Diagnostic Reference Levels in Germany - Improvements and Consequences. s.l., s.n.

Health, E. P. (2018). Guidance national diagnostic reference levels: 22 January 2016 to 14 November 2018. England Public Health.

IBRAHIM ABDULAI SAWANEH, ALBERT KAMARA, and JOSHUA HAMID
KOROMA. (2018). A computerized patient's database management system. International Journal of Computer Science and Information Technology Research, 6(2).

JERROLD T. BUSHBERG, J. ANTHONY SEIBERT, EDWIN M. LEIDHOLDT JR, and JOHN M. BOONE. (2012). The essential physics of medical imaging. Philadelphia: Wolters Kluwer.

K. Eckerman, J. Harrison, H-G. Menzel, C.H. Clement. (2012). Compendium of dose coefficients based on ICRP publication 60 . ELSEVIER.

Kruse Clemens Scott Kruse, Rishi Goswamy, Yesha Raval, and Sarah Marawi (2016). Challenges and opportunities of big data in health care: A systematic review. JMIR Medical Informatics, 4. doi:10.2196/medinform.5359

Luís A. Bastião Silva; Luís Ribeiro ; Carlos Costa ; José Luis Oliveira ; and Milton Santos. (2014, July 24). Normalizing medical imaging archives for dose quality assurance and productivity auditing. (IEEE Xplore) doi:10.1109/MeMeA.2014.6860112

Magda Bosch de Basea, Jane A. Salotti, Mark S. Pearce, Jordi Muchart, Luis Riera, Ignasi Barber, Salvador Pedraza, Marina Pardina, Antoni Capdevila, Ana Espinosa, and Elisabeth Cardis. (2015). Trends and patterns in the use of computed tomography in children and young adults in catalonia - results from the EPI-CT study. Springer. doi:10.1007/s00247-015-3434-5

Mark S Pearce, Jane A Salotti, Mark P Little, Kieran McHugh, Choonsik Lee, Kwang Pyo Kim, Nicola L Howe, Cecile M Ronckers, Preetha Rajaraman, Sir Alan W Craft, Louise Parker, Amy Berrington de González. (2012). Radiation exposure from CT scans in childhood and subsequent risk of Leukaemia and brain tumours: A retrospective cohort study. The Lancet.

Masanao Kobayashi, Yasuki Asada, Kosuke Matsubara, Tomonobu Haba, Yuta Matsunaga, Ai Kawaguchi, Kazuhiro Katada, Hiroshi Toyama, Kichiro Koshida, Ryoichi Kato, Shouichi Suzuki. (2019). Evaluation of effective dose using the k-factor of optimal scan range for CT examination. Scientific Research Publishing.

Nakada Y, Okuda Y, Tsuge T, Suzuki J, Sakamoto H, Yamamoto T, Konishi Y, Tsujimoto T, Nishiki S, Satoh T, Aoyama N, Morimoto K, Aita M, Yamashita Y, Yoshitake T, Mukai M, Yokooka Y, Yokohama N, Akahane K. (2018). Automatic acquisition of CT radiation dose data: Using the diagnostic reference level 
for radiation dose optimization. NCBI, 181(2). doi:10.1093/rpd/ncy003

Paul D Deak, Yulia Smal, and Willi A Kalender. (2010). Multisection CT protocols: sex- and age-specific conversion factors used to determine effective dose from dose-length product. National Center for Biotechnology Information

(NCBI). doi:10.1148/radiol.10100047

Premkumar Balaraman, and Kalpana Kosalram. (2013). E -hospital management \& hospital information systems - changing trends. Information Engineering and Electronic Business.

Prodromos D. Chatzoglou, Leonidas L. Fragidis, Triantafyllia Doumpa, and Vassilios. (2012). Hospital information system evaluation. 10th International Conference on Information Communication Technologies in Health. Samos Island Greece.

Rade R. Babić, Zoran Milošević, Boris Đinđić, and Gordana Stanković-Babić. (2012). Radiology information system. Professional article. doi:10.5633/amm.2012.0406

Radiologists, T. R. (2008). Radiology information systems. London: The Royal College of Radiologists.
Richard G. Kavanagh, John O'Grady, Brian W. Carey, Patrick D. McLaughlin, Siobhan B. O’Neill, Michael M. Maher, and Owen J. O'Connor. (2018). Low-dose computed tomography for the optimization of. Hindawi. Retrieved from https://www.hindawi.com/journals/grp/2018/1 $768716 /$

Richard Veit, and Burkhard Bauer. (2019). Introduction of diagnostic reference levels into diagnostic radiology in Germany. Retrieved from

https://www.researchgate.net/publication/2373 34441_Introduction_of_Diagnostic_Reference _Levels_into_Diagnostic_Radiology_in_Germ any

VALENTIN, J. (2007). The 2007 recommendations of the international commission on radiological protection. ELSEVIER.

Walter Huda, Kent M. Ogden, and Mohammad R. Khorasani. (2008). Converting dose-length product to effective dose at CT. Radiology.

Yousef Mehdipour, and Hamideh Zerehkafi. (2013). Hospital information system (HIS): At a glance. Asian Journal of Computer and Information Systems, 1(2). 